\title{
Дискуссия
}

Уважсаемые читатели!

Редакционная коллегия журнала решила возобновить рубрику «Дискуссия», в которой намерена публиковать статьи с неоднозначными отзывами рецензентов. Надеемся, что обсуждение таких работ будет способствовать установлению научной истины.

В этом номере мы публикуем статью Льва Николаевича Мизеровского, профессора Ивановского государственного химико-технологического университета, в которой он излагает свои взгляды на жидкостное равновесие. Полученные выводы вытекают из его более чем 50-летнего опыта в работе с гомогенными бинарными жидкими смесями.

Приведенные в работе положения являются дискуссионными, и редакция надеется получить отклики на выводы, изложенные в статье.

УДК 544.353.21

DOI: $10.6060 / \mathrm{rcj} .2021652 .11$

\section{ЖИДКОСТНОЕ РАВНОВЕСИЕ}

\section{Л.Н. Мизеровский}

ЛЕВ НИКОЛАЕВИЧ МИЗЕРОВСКИЙ - д.х.н., профессор Ивановского государственного химико-технологического университета.E-mail: Inmizerovskii2303@mail.ru.

Ивановский государственный химико-технологический университет, 153000, г. Иваново, Шереметевский пр., д. 7.

Показано, что химические потенцииаль компонентов бинарных жидких смесей, образуюших ветви бинодали жидкостного равновесия действительно численно равны, но имеют противоположные (+ и -) знаки и размерность Дюсмоль, а условием этого равновесия является численное равенство температуре, при которой равновесие реализуется, отношений изменений энтальпии к изменениям энтропии сосуществуюших пар бинарных смесей при их превращении из гетерогенных в гомогенные.

Ключевые слова: жидкость, фаза, энергия Гиббса, химический потенциал, равновесие.

\section{LIQUID EQUILIBRIUM}

\section{N. Mizerovskiy}

Ivanovo State University of Chemistry and Technology, Russian Federation, 153000, Ivanovo, Sheremetev av., 7

It has been shown that chemical potentials of components in coexisting liquid phases are in fact numerically equal, but have opposite signs and dimension J/mole. The thermodynamic condition for a phase equilibrium is the numerical equality to the temperature under which equilibrium is realized, relations of enthalpy changes to entropy changes during the conversion process of a binary mixture from heterogeneous to a homogeneous one.

Keywords: liquid, phase, Gibbs energy, chemical potential, equilibrium. 
Термином жидкостное равновесия принято обозначать ситуацию, когда гомогенная жидкая смесь при охлаждении или нагревании разделяется на два слоя (фазы), соотношение концентраций компонентов которых зависит от температуры.

Фрагменты диаграммы, отражающей эту ситуацию, могут быть построены по результатам определения состава фаз, образующихся при расслаивании смесей различного состава. Диаграмму же, отражающую фазовое равновесие в бинарных жидких смесях как таковую (рисунок), можно построить только по результатам определения состава фаз, самопроизвольно образующихся при ступенчатом охлаждении смеси эквимольного (критического) состава.

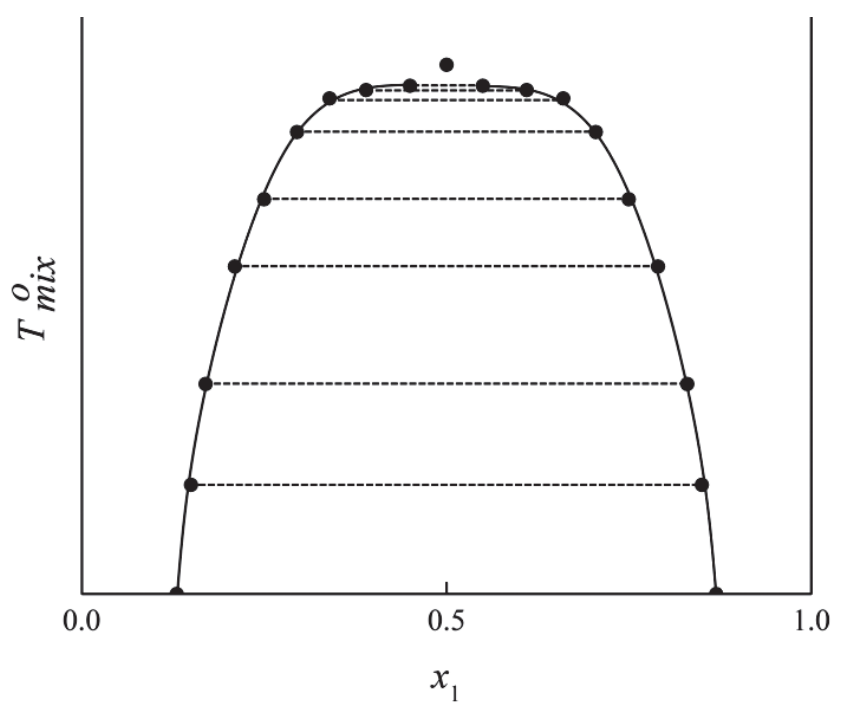

Рис. Бинодаль жидкостного равновесия

Равенство количеств молекул двух жидкостей является в этом случае гарантией того, что различие в составах сосуществующих фаз обсуловлено исключительно изменением температуры.

Особенность ветвей этой диаграммы заключается в том, что они в принципе не могут перейти одна в другую, так как начинаются с первых двух фаз, самопроизвольно образующихся при расслаивании смеси эквимольного состава.

Поэтому общепринятая [1-6] практика представления этих диаграмм в виде куполообразной линии противоречит их термодинамическому смыслу.

В отличие от ветвей диаграмм, отражающих температурные зависимости взаимной растворимости жидкостей, ветви бинодали не имеют «собственного» термодинамического смысла. Они лишь отражают составы пар бинарных смесей, сосуществующих в условиях термодинамического равновесия в некотором температурном интервале. Следовательно, эти ветви нельзя воспроизвести, их можно только построить.

Энергия Гиббса гомогенной смеси эквимольного (критического) состава при наименьшей температуре ее превразения из гетерогенной в гомогенную равна разности:

$$
G_{m i x}^{c r}=H_{m i x}^{c r}-T_{m i x} S_{m i x}^{c r}-\Delta G_{m i x}^{c r}
$$

а при любой другой температуре - разности

$$
G_{m i x}^{c r}=H_{m i x}^{c r}-T_{m i x} S_{m i x}^{c r}
$$

Поэтому по отношению к парам бинарных смесей самопроизвольно образующимся в результате расслаивания смеси эквимольного состава, должны быть справедливы равенства: первая фаза:

$$
\begin{gathered}
\Delta G_{m i x}^{I}=\Delta H_{m i x}^{I}-T_{m i x} \Delta S_{m i x}^{I}=0 \\
\Delta H_{m i x}^{I}=T_{m i x} \Delta S_{m i x}^{I}
\end{gathered}
$$

вторая фаза:

$$
\begin{gathered}
\Delta G_{m i x}^{I I}=\Delta H_{m i x}^{I I}-T_{m i x} \Delta S_{m i x}^{I I}=0 \\
\Delta H_{m i x}^{I I}=T_{m i x} \Delta S_{m i x}^{I I}
\end{gathered}
$$

из которых следует соотношение

$$
\left(\frac{\Delta H_{m i x}}{\Delta S_{\text {mix }}}\right)^{I}=\left(\frac{\Delta H_{\text {mix }}}{\Delta S_{\text {mix }}}\right)^{I I}=T_{\text {mix }}
$$

отражающее термодинамическое условие фазового равновесия в бинарных жидких смесях.

Сама же смесь критического состава характеризуется неравенствами:

$$
\begin{gathered}
\Delta G_{m i x}^{c r}=\Delta H_{m i x}^{c r}-T_{m i x} \Delta S_{m i x}^{c r}<0 \\
\Delta H_{m i x}^{c r}<T_{m i x} \Delta S_{m i x}^{c r}
\end{gathered}
$$

подтверждающими, что она может быть как «особенной» $\left(\Delta \mathrm{H}_{\text {mix }}=0\right)$, так и обычной $\left(\Delta \mathrm{H}_{\text {mix }}<0\right)$, но в любом случае термодинамически неэквивалентной образующимся при ее самопроизвольном расслаивании бинарным смесям, образующим в совокупности ветви бинодали.

Поскольку эти смеси отвечают условию $\Delta \mathrm{H}_{\text {mix }}=0$, к ним применимы соотношения:

$$
G^{I}=G_{1}^{o} x_{1}^{I}+G_{2}^{o} x_{2}^{I}=G_{2}^{o}+\left(G_{1}^{o}-G_{2}^{o}\right) x_{1}^{I}
$$




$$
\begin{gathered}
G^{I I}=G_{1}^{o} x_{1}^{I I}+G_{2}^{o} x_{2}^{I I}=G_{2}^{o}+\left(G_{1}^{o}-G_{2}^{o}\right) x_{1}^{I I} \\
\mu_{1}^{I}=\left(\frac{\partial G}{\partial x_{1}}\right)_{T, p}^{I}=\left(G_{1}^{o}-G_{2}^{o}\right) \\
\mu_{1}^{I I}=\left(\frac{\partial G}{\partial x_{1}}\right)_{T, p}^{I I}=\left(G_{1}^{o}-G_{2}^{o}\right) \\
\mu_{2}^{I}=\left(\frac{\partial G}{\partial x_{1}}\right)_{T, p}^{I I}=-\left(G_{1}^{o}-G_{2}^{o}\right) \\
\mu_{2}^{I I}=\left(\frac{\partial G}{\partial x_{1}}\right)_{T, p}^{I I}=-\left(G_{1}^{o}-G_{2}^{o}\right)
\end{gathered}
$$

подтверждающие справедливость общепринятой точки зрения относительно численного равенства первых производных энергий Гиббса бинарных смесей по мольной доле любого из компонентов, которые приято характеризовать термином «химический потенциал» компонента.

Равенство этих производных (углов наклона касательных к ветвям бинодали в любой их точке) означает, что ветви бинодали симметричны, как это показано на рисунке.

Однако само по себе равенство углов наклона этих касательных не может рассматриваться в качестве параметра, отражающего термодинамическое условие фазового равновесия.

Это условие отражает равенство (7).

Изложенное дает основание утверждать:

1. Гомогенные бинарные жидкие смеси могут находиться в двух термодинамических состояниях: метастабильном, отвечающем равенству

$$
\Delta H_{m i x}=T_{m i x} \Delta S_{m i x}
$$

И термодинамически устойчивом, отвечающем неравенству

$$
\Delta H_{m i x}<T_{m i x} \Delta S_{m i x}
$$

2. Химические потенциалы компонентов имеют размерность Дж/моль, их численные значения в сосуществующих фазах численно равны, но имеют противоположные (+ и -) знаки.

3. Ветви бинодали отражают факт сосуществования в условиях термодинамического равновесия пар бинарных смесей, особенных тем, что отношения изменений энтальпии к изменениям энтропии при превращении их из гетерогенных в гомогенные численно равны температуре, при которой эти превращения происходят.

4. Термодинамическое условие фазового равновесия в бинарных жидких смесях отражает равенство (7).

\section{Лumepamypa}

1. Курс физической химии Т. 1. / Под ред. Я.И. Герасимова М.: Госхимиздат, 1963. 398 с.

2. Жуховиикий А.А., Шваримман А.А. Физическая химия. М.: Металлургия, 1968. 131 с.

3. Киреев В.A. Курс физической химии. М.: Химия, $1975.397 \mathrm{c}$.

4. Эткинс П.Э. Физическая химия. М.: Мир, 1980. 319 с.

5. Atkins P.W. Physical chemistry. Oxford Press. 1978.

6. Физическая химия. Теоретическое и практическое руководство / Под. ред. Б.Н. Никольского. Л.: Химия, 1987. 291 с.

7. Физическая химия. Строение вещества. Термодинамика / Под ред. К.С. Краснова. М.: Высшая школа, 2001. 442 с.

\section{References}

1. Kurs fizicheskoy khimii. T 1. / Pod red. Ya. I. Gerasimova. M.: Goschimizdat, 1963. 398 s.

2. Zhukhovitskiy A.A., Shvartsman A.A. Fizicheskaya khimiya. M.: Metallurgiya, 1968. $131 \mathrm{~s}$.

3. Kireev V.A. Kurs fizicheskoy khimii. M.: Khimiya, 1975. 397 c.

4. Etkins P.E. Fizicheskaya khimiya. M.: Mir, 1980. 319 c.

5. Atkins P.W. Physical chemistry. Oxford Press. 1978.

6. Fizicheskaya khimiya. Teoreticheskoye I prakticheskoye rukovodstvo / Pod. red. B.N. Nikolskogo. L.: Khimiya, 1987. $291 \mathrm{~s}$.

7. Fizicheskaya khimiya. Stroyeniye veshchestva. Termodinamika / Pod. red. K.S. Krasnova. M.: Vysshaya shkola, 2001. $442 \mathrm{~s}$. 